\title{
The fourth-order Hermitian Toeplitz determinant for convex functions
}

\author{
Adam Lecko $^{1}\left[\right.$ [ Young Jae $\mathrm{Sim}^{2} \cdot$ Barbara Śmiarowska $^{1}$ \\ Received: 23 October 2019 / Revised: 23 October 2019 / Accepted: 3 August 2020 / \\ Published online: 12 August 2020 \\ (c) The Author(s) 2020
}

\section{Abstract}

The sharp bounds for the fourth-order Hermitian Toeplitz determinant over the class of convex functions are computed.

Keywords Hermitian Toeplitz determinant . Univalent functions · Convex functions . Carathéodory class

Mathematics Subject Classification 30C45 - 30C50

\section{Introduction}

Let $\mathcal{H}$ be the class of analytic functions in $\mathbb{D}:=\{z \in \mathbb{C}:|z|<1\}, \mathcal{A}$ be its subclass normalized by $f(0):=0, f^{\prime}(0):=1$, that is, functions of the form

$$
f(z)=\sum_{n=1}^{\infty} a_{n} z^{n}, \quad a_{1}:=1, \quad z \in \mathbb{D}
$$

and $\mathcal{S}$ be the subclass of $\mathcal{A}$ of univalent functions. Let $\mathcal{S}^{c}$ denote the subclass of $\mathcal{S}$ of convex functions, that is, univalent functions $f \in \mathcal{A}$ such that $f(\mathbb{D})$ is a convex domain in $\mathbb{C}$. By the well-known result of Study [11] (see also [5, p. 42]), a function

\footnotetext{
$凶 \quad$ Adam Lecko

alecko@matman.uwm.edu.pl

Young Jae Sim

yjsim@ks.ac.kr

Barbara Śmiarowska

b.smiarowska@matman.uwm.edu.pl

1 Department of Complex Analysis, Faculty of Mathematics and Computer Science, University of Warmia and Mazury in Olsztyn, ul. Słoneczna 54, 10-710 Olsztyn, Poland

2 Department of Mathematics, Kyungsung University, Busan 48434, Korea
}

(Dirkhäuser 
$f$ is in $\mathcal{S}^{c}$ if and only if

$$
\operatorname{Re}\left\{1+\frac{z f^{\prime \prime}(z)}{f^{\prime}(z)}\right\}>0, \quad z \in \mathbb{D} \text {. }
$$

Given $q, n \in \mathbb{N}$, the Hermitian Toeplitz matrix $T_{q, n}(f)$ of $f \in \mathcal{A}$ of the form (1) is defined by

$$
T_{q, n}(f):=\left[\begin{array}{cccc}
a_{n} & a_{n+1} & \ldots & a_{n+q-1} \\
\bar{a}_{n+1} & a_{n} & \ldots & a_{n+q-2} \\
\vdots & \vdots & \vdots & \vdots \\
\bar{a}_{n+q-1} & \bar{a}_{n+q-2} & \ldots & a_{n}
\end{array}\right]
$$

where $\bar{a}_{k}:=\overline{a_{k}}$. Let $\left|T_{q, n}(f)\right|$ denote the determinant of $T_{q, n}(f)$. In particular, the third Toeplitz determinant $\left|T_{3,1}(f)\right|$ is given by

$$
\left|T_{3,1}(f)\right|=\left|\begin{array}{ccc}
1 & a_{2} & a_{3} \\
\bar{a}_{2} & 1 & a_{2} \\
\bar{a}_{3} & \bar{a}_{2} & 1
\end{array}\right|=1+2 \operatorname{Re}\left(a_{2}^{2} \bar{a}_{3}\right)-2\left|a_{2}\right|^{2}-\left|a_{3}\right|^{2}
$$

and the fourth Toeplitz determinant $\left|T_{4,1}(f)\right|$ is given by

$$
\begin{aligned}
\left|T_{4,1}(f)\right|= & \left|\begin{array}{llll}
a_{1} & a_{2} & a_{3} & a_{4} \\
\bar{a}_{2} & a_{1} & a_{2} & a_{3} \\
\bar{a}_{3} & \bar{a}_{2} & a_{1} & a_{2} \\
\bar{a}_{4} & \bar{a}_{3} & \bar{a}_{2} & a_{1}
\end{array}\right| \\
= & 1-2 \operatorname{Re}\left(a_{2}^{3} \bar{a}_{4}\right)+4 \operatorname{Re}\left(a_{2}^{2} \bar{a}_{3}\right)-2 \operatorname{Re}\left(a_{2} \bar{a}_{3}^{2} a_{4}\right) \\
& +4 \operatorname{Re}\left(a_{2} a_{3} \bar{a}_{4}\right)+\left|a_{2}\right|^{4}-3\left|a_{2}\right|^{2}+\left|a_{3}\right|^{4}-2\left|a_{3}\right|^{2} \\
& +\left|a_{2}\right|^{2}\left|a_{4}\right|^{2}-2\left|a_{2}\right|^{2}\left|a_{3}\right|^{2}-\left|a_{4}\right|^{2} .
\end{aligned}
$$

In recent years a lot of papers has been devoted to the estimation of determinants built with using coefficients of functions in the class $\mathcal{A}$ or its subclasses. Hankel matrices i.e., square matrices which have constant entries along the reverse diagonal (see e.g., [3] with further references), and the symmetric Toeplitz determinant (see [1]) are of particular interest.

For this reason looking on the interest of specialists in [4] and [7] the study of the Hermitian Toeplitz determinants on the class $\mathcal{A}$ or its subclasses has begun. Hermitian Toeplitz matrices play an important role in functional analysis, applied mathematics as well as in physics and technical sciences.

In [4] the conjecture that the sharp inequalities $0 \leq\left|T_{q, 1}(f)\right| \leq 1$ for all $q \geq 2$, holds over the class $\mathcal{S}^{c}$ was proposed and was confirmed for $q=2$ and $q=3$. The purpose of this paper is to prove this conjecture for $q=4$. 
Let $\mathcal{P}$ be the class of all $p \in \mathcal{H}$ of the form

$$
p(z)=1+\sum_{n=1}^{\infty} c_{n} z^{n}, \quad z \in \mathbb{D}
$$

having a positive real part in $\mathbb{D}$.

The key to the proof of the main result is the following lemma. It contains the well-known formula for $c_{2}$ (see e.g., [10, p. 166]) and the formula for $c_{3}$ due to Libera and Zlotkiewicz [8,9].

Lemma 1 If $p \in \mathcal{P}$ is of the form (5) with $c_{1} \geq 0$, then

$$
2 c_{2}=c_{1}^{2}+\left(4-c_{1}^{2}\right) \zeta
$$

and

$$
4 c_{3}=c_{1}^{3}+\left(4-c_{1}^{2}\right) c_{1} \zeta(2-\zeta)+2\left(4-c_{1}^{2}\right)\left(1-|\zeta|^{2}\right) \eta
$$

for some $\zeta, \eta \in \overline{\mathbb{D}}:=\{z \in \mathbb{C}:|z| \leq 1\}$

\section{Main result}

In [4] the sharp bounds for the Hermitian-Toeplitz determinants of the second and third-order for the class of convex functions of order $\alpha$ were computed. In particular for $\alpha=0$, the results obtained are reduced for the class of convex functions, namely, $0 \leq\left|T_{2,1}(f)\right| \leq 1$ and $0 \leq\left|T_{3,1}(f)\right| \leq 1$ when $f \in \mathcal{S}^{c}$. Both results suggested the conjecture that $0 \leq\left|T_{q, 1}(f)\right| \leq 1$ for every $q \geq 2$. In this paper, this conjecture was confirmed for $q=4$. For the sake of consistency, we also provide a short proof of the case $q=3$.

Theorem 1 If $f \in \mathcal{S}^{c}$, then

$$
0 \leq\left|T_{3,1}(f)\right| \leq 1
$$

Both inequalities are sharp with equalities attained by

$$
f(z)=\frac{z}{1-z}, \quad z \in \mathbb{D}
$$

and by the identity, respectively.

Proof Let $f \in \mathcal{S}^{c}$ be the form (1). Then by (2),

$$
f^{\prime}(z)+z f^{\prime \prime}(z)=p(z) f^{\prime}(z), \quad z \in \mathbb{D},
$$


for some $p \in \mathcal{P}$ of the form (5). Substituting the series (1) and (5) into (10) by equating the coefficients we get

$$
a_{2}=\frac{1}{2} c_{1}, \quad a_{3}=\frac{1}{6}\left(c_{1}^{2}+c_{2}\right), \quad a_{4}=\frac{1}{24}\left(c_{1}^{3}+3 c_{1} c_{2}+2 c_{3}\right) .
$$

Noting that the class $\mathcal{S}^{c}$ and $\left|T_{3,1}(f)\right|$ are rotationally invariant, we may assume that $c:=c_{1}$ with $c \in[0,2]$ ([2], see also [6, Vol. I, page 80, Theorem 3]), i.e., by (11), $a_{2} \in[0,1]$. Then by (3) and (11) we have

$$
\begin{aligned}
\left|T_{3,1}(f)\right| & =1+2 a_{2}^{2} \operatorname{Re} a_{3}-2 a_{2}^{2}-\left|a_{3}\right|^{2} \\
& =\frac{1}{72}\left(72-36 c^{2}+4 c^{4}+2 c^{2} \operatorname{Re} c_{2}-2\left|c_{2}\right|^{2}\right) .
\end{aligned}
$$

Now by using (6) and (7) we get

$$
0 \leq\left|T_{3,1}(f)\right|=\frac{1}{144}\left(4-c^{2}\right)^{2}\left(9-|\zeta|^{2}\right) \leq 1
$$

which shows (8).

It is clear that the function (9) and the identity make the results sharp.

We will now estimate the fourth-order Toeplitz determinant $\left|T_{4,1}(f)\right|$ for $f \in \mathcal{S}^{c}$.

Theorem 2 If $f \in \mathcal{S}^{c}$, then

$$
0 \leq\left|T_{4,1}(f)\right| \leq 1
$$

Both inequalities are sharp with equalities attained by the function (9) and the identity, respectively.

Proof Assuming as in the proof of Theorem 1 that $c:=c_{1}$ with $c \in[0,2]$, so $a_{2} \in[0,1]$, from (4) and (11) we obtain

$$
\begin{aligned}
\left|T_{4,1}(f)\right|= & +a_{2}^{4}-3 a_{2}^{2}+4 a_{2}^{2} \operatorname{Re} a_{3}-2 a_{2}^{2}\left|a_{3}\right|^{2}-2 a_{2}^{3} \operatorname{Re} a_{4}+a_{2}^{2}\left|a_{4}\right|^{2} \\
& +4 a_{2} \operatorname{Re}\left(a_{3} \bar{a}_{4}\right)-2 a_{2} \operatorname{Re}\left(a_{3}^{2} \bar{a}_{4}\right)-2\left|a_{3}\right|^{2}+\left|a_{3}\right|^{4}-\left|a_{4}\right|^{2} \\
= & \frac{1}{20736}\left(20736-15552 c^{2}+3600 c^{4}-252 c^{6}+c^{8}+1152 c^{2} \operatorname{Re} c_{2}\right. \\
& -288 c^{4} \operatorname{Re} c_{2}+64 c^{4}\left(\operatorname{Re} c_{2}\right)^{2}-2 c^{6} \operatorname{Re} c_{2}+252 c^{2}\left|c_{2}\right|^{2} \\
& -31 c^{4}\left|c_{2}\right|^{2}+16\left|c_{2}\right|^{4}-1152\left|c_{2}\right|^{2}-24 c^{4} \operatorname{Re} c_{2}^{2} \\
& -8 c^{2}\left|c_{2}\right|^{2} \operatorname{Re} c_{2}-12 c^{5} \operatorname{Re} c_{3}+144 c \operatorname{Re}\left(c_{2} \bar{c}_{3}\right) \\
& \left.+12 c^{3} \operatorname{Re}\left(c_{2} \bar{c}_{3}\right)-48 c \operatorname{Re}\left(c_{2}^{2} \bar{c}_{3}\right)-144\left|c_{3}\right|^{2}+36 c^{2}\left|c_{3}\right|^{2}\right) .
\end{aligned}
$$


Hence by using (6) and (7) after tedious computation we get

$$
\begin{aligned}
\left|T_{4,1}(f)\right|= & \frac{1}{82944}\left(4-c^{2}\right)^{3}\left[1296-9\left(c^{2}+32\right)|\zeta|^{2}\right. \\
& +6 c^{2}|\zeta|^{2} \operatorname{Re} \zeta+\left(16-c^{2}\right)|\zeta|^{4}-36 c\left(1-|\zeta|^{2}\right) \operatorname{Re}(\zeta \bar{\eta}) \\
& \left.+12 c\left(1-|\zeta|^{2}\right) \operatorname{Re}\left(\zeta^{2} \bar{\eta}\right)-36\left(1-|\zeta|^{2}\right)^{2}|\eta|^{2}\right]
\end{aligned}
$$

A. Suppose that $\zeta=0$. Then

$$
0 \leq\left|T_{4,1}(f)\right|=\frac{1}{64}\left(4-c^{2}\right)^{3} \leq 1 .
$$

Suppose that $\eta=0$. Then

$$
\begin{aligned}
\left|T_{4,1}(f)\right|= & \frac{1}{82944}\left(4-c^{2}\right)^{3}\left[1296-9\left(c^{2}+32\right)|\zeta|^{2}\right. \\
& \left.+6 c^{2}|\zeta|^{2} \operatorname{Re} \zeta+\left(16-c^{2}\right)|\zeta|^{4}\right]
\end{aligned}
$$

We have

$$
\begin{aligned}
& 1296-9\left(c^{2}+32\right)|\zeta|^{2}+6 c^{2}|\zeta|^{2} \operatorname{Re} \zeta+\left(16-c^{2}\right)|\zeta|^{4} \\
& \geq 1296-9\left(c^{2}+32\right)|\zeta|^{2}-6 c^{2}|\zeta|^{3}+\left(16-c^{2}\right)|\zeta|^{4} \\
& =1296-288|\zeta|^{2}+16|\zeta|^{4}-|\zeta|^{2}(3+|\zeta|)^{2} c^{2} \\
& \geq 1296-288|\zeta|^{2}+16|\zeta|^{4}-4|\zeta|^{2}(3+|\zeta|)^{2} \\
& =1296-324|\zeta|^{2}-24|\zeta|^{3}+12|\zeta|^{4} \geq 960, \quad 0 \leq|\zeta| \leq 1 .
\end{aligned}
$$

Hence and from (15) it follows that $\left|T_{4,1}(f)\right| \geq 0$.

We have

$$
\begin{aligned}
& 1296-9\left(c^{2}+32\right)|\zeta|^{2}+6 c^{2}|\zeta|^{2} \operatorname{Re} \zeta+\left(16-c^{2}\right)|\zeta|^{4} \\
& \leq 1296-9\left(c^{2}+32\right)|\zeta|^{2}+6 c^{2}|\zeta|^{3}+\left(16-c^{2}\right)|\zeta|^{4} \\
& =1296-288|\zeta|^{2}+16|\zeta|^{4}-|\zeta|^{2}(3-|\zeta|)^{2} c^{2} \\
& \leq 1296-288|\zeta|^{2}+16|\zeta|^{4} \leq 1296, \quad 0 \leq|\zeta| \leq 1 .
\end{aligned}
$$

Hence and from (15) it follows that $\left|T_{4,1}(f)\right| \leq 1$.

B. Let now $\zeta, \eta \in \overline{\mathbb{D}} \backslash\{0\}$. By setting $\zeta:=x \mathrm{e}^{\mathrm{i} \theta}, \eta:=y \mathrm{e}^{\mathrm{i} \psi}, x, y \in(0,1], \theta, \psi \in$ $[0,2 \pi)$, from (14) we get

$$
\left|T_{4,1}(f)\right|=\frac{1}{82944}\left(4-c^{2}\right)^{3} F(c, x, y, \theta, \psi),
$$


where

$$
\begin{aligned}
F(c, x, y, \theta, \psi):= & 1296-9\left(c^{2}+32\right) x^{2}+6 c^{2} x^{3} \cos \theta+\left(16-c^{2}\right) x^{4} \\
& -36 c\left(1-x^{2}\right) x y \cos (\theta-\psi)+12 c\left(1-x^{2}\right) x^{2} y \cos (2 \theta-\psi) \\
& -36\left(1-x^{2}\right)^{2} y^{2}
\end{aligned}
$$

For $c \in[0,2]$ and $x, y \in(0,1]$ we have

$$
G(c, x, y) \leq F(c, x, y, \theta, \psi) \leq H(c, x, y)
$$

where for $x, y \in[0,1]$,

$$
\begin{aligned}
& G(c, x, y):=F(c, x, y, \pi, \pi) \\
& =1296-9\left(c^{2}+32\right) x^{2}-6 c^{2} x^{3}+\left(16-c^{2}\right) x^{4} \\
& \quad-12 c\left(1-x^{2}\right)(3+x) x y-36\left(1-x^{2}\right)^{2} y^{2}
\end{aligned}
$$

and

$$
\begin{aligned}
H(c, x, y):= & 1296-9\left(c^{2}+32\right) x^{2}+6 c^{2} x^{3}+\left(16-c^{2}\right) x^{4} \\
& +12 c\left(1-x^{2}\right)(3+x) x y-36\left(1-x^{2}\right)^{2} y^{2}
\end{aligned}
$$

C. Let us observe that

$$
\begin{aligned}
G(c, x, y)= & 1296-9\left(c^{2}+32\right) x^{2}-6 c^{2} x^{3}+\left(16-c^{2}\right) x^{4} \\
& -12 c\left(1-x^{2}\right)(3+x) x y-36\left(1-x^{2}\right)^{2} y^{2} \\
\geq & 1296-324 x^{2}-24 x^{3}-24\left(1-x^{2}\right)(3+x) x-36\left(1-x^{2}\right)^{2} \\
\geq & 1296-480=816, \quad c \in[0,2], x, y \in(0,1] .
\end{aligned}
$$

Hence and from (16) with (17) it follows that

$$
\begin{aligned}
\left|T_{4,1}(f)\right| & =\frac{1}{82944}\left(4-c^{2}\right)^{3} F(c, x, y, \theta, \psi) \\
& \geq \frac{1}{82944}\left(4-c^{2}\right)^{3} G(c, x, y) \geq \frac{17}{1728}\left(4-c^{2}\right)^{3} \geq 0, \quad c \in[0,2],
\end{aligned}
$$

which together with part A shows the lower bound in (12).

D. Now will discuss the upper bound of $\left|T_{4,1}(f)\right|$.

Let first $x=1$. Then for $c \in[0,2]$ and $y \in(0,1]$,

$$
H(c, 1,1)=H(c, 1, y)=1024-4 c^{2} \leq 1024, \quad c \in[0,2] .
$$

Let now $x \in(0,1)$. Then

$$
y_{w}:=\frac{c x(x+3)}{6\left(1-x^{2}\right)} \geq 0, \quad-36\left(1-x^{2}\right)^{2}<0 .
$$


Therefore we consider two cases.

D1. Assume that $y_{w}<1$, i.e., equivalently that $x \in\left(0, x_{1}(c)\right)$, where

$$
x_{1}(c):=\frac{-3 c+\sqrt{9 c^{2}+24 c+144}}{2(c+6)}, \quad c \in[0,2] .
$$

Note that $x_{1}(c) \leq 1$ for all $c \in[0,2]$ and $x_{1}(0)=1$. Let $\Delta_{1}:=\{(c, x): 0 \leq c \leq$ $\left.2,0 \leq x \leq x_{1}(c)\right\}$. We have

$$
H(c, x, y) \leq H\left(c, x, y_{w}\right)=h(c, x), \quad(c, x) \in \Delta_{1}, y \in(0,1]
$$

where

$$
h(c, x):=1296-288 x^{2}+12 c^{2} x^{3}+16 x^{4}, \quad(c, x) \in \Delta_{1} .
$$

(i) On the vertices of $\Delta_{1}$,

$$
\begin{aligned}
& h(0,0)=1296, \quad h\left(0, x_{1}(0)\right)=h(0,1)=1296, \quad h(2,0)=1296, \\
& h\left(2, x_{1}(2)\right)=h\left(2, \frac{1}{8}(\sqrt{57}-3)\right)=\frac{9}{32}(3461+113 \sqrt{57}) \approx 1213.349 .
\end{aligned}
$$

(ii) On the side $x=0$,

$$
h(c, 0)=1296, \quad c \in(0,2) .
$$

(iii) On the side $x=x_{1}(c)$ for $c \in(0,2)$,

$$
\begin{aligned}
& h\left(c, x_{1}(c)\right) \\
= & \frac{18}{(c+6)^{4}}\left(-9 c^{6}-72 c^{5}-180 c^{4}+216 c^{3}+11840 c^{2}+52224 c+73728\right. \\
& \left.+\left(3 c^{5}+20 c^{4}+36 c^{3}+344 c^{2}+768 c\right) \sqrt{9 c^{2}+24 c+144}\right)=: \gamma(c) .
\end{aligned}
$$

We will show that $\gamma$ increasis. Note that

$$
\gamma^{\prime}(c)=\frac{-36\left(\varrho_{1}(c)+\varrho_{2}(c) \sqrt{9 c^{2}+24 c+144}\right)}{(c+6)^{5} \sqrt{9 c^{2}+24 c+144}}>0, \quad c \in(0,2),
$$

is equivalent to

$$
\varrho_{1}(c)+\varrho_{2}(c) \sqrt{9 c^{2}+24 c+144}<0, \quad c \in(0,2),
$$


where for $t \in \mathbb{R}$,

$$
\varrho_{1}(t):=-27 t^{7}-630 t^{6}-4224 t^{5}-15084 t^{4}-55800 t^{3}-77472 t^{2}-214272 t-331776
$$

and

$$
\varrho_{2}(t):=9 t^{6}+198 t^{5}+1080 t^{4}+2268 t^{3}+9896 t^{2}+7296 t-9216 .
$$

Since $\varrho_{1}^{\prime}$ has only two real zeros at $c \approx-12.8436$ and $c \approx-5.43834$, and $\varrho_{1}^{\prime}(0)<0$, so $\varrho_{1}$ decreases on $(0,2)$. This with $\varrho_{1}(0)<0$ yields

$$
\varrho_{1}(c)<0, \quad c \in(0,2) .
$$

Since $\varrho_{2}^{\prime}>0$ for $c \in[0,2]$, so $\varrho_{2}$ increases from $\varrho_{2}(0)=-9216$ to $\varrho_{2}(2)=87296$. Thus $\varrho_{2}(c) \leq 0$ for $c \in\left(0, c^{\prime}\right]$ and $\varrho_{2}(c)>0$ for $c \in\left(c^{\prime}, 2\right)$, where $c^{\prime} \approx 0.627225$ is the unique zero of $\gamma_{2}$ in $(0,2)$.

Hence and from (20) it follows that the inequality (19) occurs for $c \in\left(0, c^{\prime}\right]$.

Note now that for $c \in\left(c^{\prime}, 2\right)$ we have

$$
\varrho_{1}^{2}(c)>\left(9 c^{2}+24 c+144\right) \varrho_{2}^{2}(c) .
$$

Indeed, the above inequality is equivalent to

$$
48(c+6)^{5} \varrho_{3}(c)<0, \quad c \in\left(c^{\prime}, 2\right),
$$

where

$\varrho_{3}(t):=9 t^{6}-30 t^{5}-4248 t^{4}-31392 t^{3}-70272 t^{2}-208896 t-262144, \quad t \in \mathbb{R}$.

As easy to see $\varrho_{3}^{\prime}(c)<0$ for $c \in(0.6,2)$, so $\varrho_{3}$ decreases on $(0.6,2)$, and therefore on $\left(c^{\prime}, 2\right)$. This and $\varrho_{3}(0.6)<0$ yields

$$
\varrho_{3}(c)<0, \quad c \in\left(c^{\prime}, 2\right),
$$

which confirms (21), (22), and ends the proof of (19) so of (18).

Summarizing,

$$
h\left(c, x_{1}\right)=\gamma(c) \leq \gamma(2)<1296, \quad c \in(0,2) .
$$

(iv) When $c=0$, then $x_{1}(0)=1$ and

$$
h(0, x)=1296-288 x^{2}+16 x^{4} \leq 1296, \quad x \in[0,1) .
$$

(v) When $c=2$, then

$$
h(2, x)=1296-288 x^{2}+48 x^{3}+16 x^{4} \leq 1296, \quad x \in\left(0, x_{1}(2)\right) .
$$


Indeed, as easy to see the function $\left(0, x_{1}(2)\right) \ni x \mapsto H_{2}(2, x)$ decreases.

(vi) It remains to consider the interior of $\Delta_{1}$. Since the system of equations

$$
\left\{\begin{array}{l}
24 c x^{3}=0 \\
-576 x+36 c^{2} x^{2}+64 x^{3}=0
\end{array}\right.
$$

has solutions $\left(c_{i}, x_{i}\right), i=1,2,3$, only when

$$
\left\{\begin{array} { l } 
{ c _ { 1 } = 0 } \\
{ x _ { 1 } = 0 }
\end{array} \quad \left\{\begin{array} { l } 
{ c _ { 2 } = 0 } \\
{ x _ { 2 } = - 3 }
\end{array} \quad \left\{\begin{array}{l}
c_{3}=0 \\
x_{3}=3
\end{array}\right.\right.\right.
$$

so $h$ has no critical points in the interior of $\Delta_{1}$.

D2. Assume that $y_{w} \geq 1$, i.e., equivalently that $x \in\left[x_{1}(c), 1\right]$. Let $\Delta_{2}:=\{(c, x)$ : $\left.0 \leq c \leq 2, x_{1}(c) \leq x \leq 1\right\}$. Then

$$
H(c, x, y) \leq H(c, x, 1)=g(c, x), \quad(c, x) \in \Delta_{2}, y \in(0,1],
$$

where for $(c, x) \in \Delta_{2}$,

$g(c, x):=1260+36 c x-3\left(3 c^{2}-4 c+72\right) x^{2}+6\left(c^{2}-6 c\right) x^{3}-\left(c^{2}+12 c+20\right) x^{4}$.

(i) On the vertices of $\Delta_{2}$,

$$
\begin{aligned}
& g\left(0, x_{1}(0)\right)=g(0,1)=1024, \quad g(2,1)=1008 \\
& g\left(2, x_{1}(2)\right)=g(2,(\sqrt{57}-3) / 8)=\frac{9}{32}(3461+113 \sqrt{57}) \approx 1213.349 .
\end{aligned}
$$

(ii) On the side $x=x_{1}(c)$ we have the case B1(iii).

(iii) On the side $x=1$,

$$
g(c, 1)=-4 c^{2}+1024 \leq 1024, \quad c \in(0,2) .
$$

(iv) On the side $c=2$,

$$
g(2, x)=-48 x^{4}-48 x^{3}-228 x^{2}+72 x+1260, \quad x \in[(\sqrt{57}-3) / 8,1) .
$$

Since $-192 x^{3}-144 x^{2}-456 x+72=0$ if and only if $x \approx 0.14944$, so the function

$$
[(\sqrt{57}-3) / 8,1) \ni x \mapsto H_{3}(2, x)
$$

is decreasing. Therefore

$$
g(2, x) \leq g(2,(\sqrt{57}-3) / 8) \approx 1213.349, \quad x \in[(\sqrt{57}-3) / 8,1) .
$$


(v) It remains to consider the interior of $\Delta_{2}$. The system of equations

$$
\begin{cases}36 x-6(3 c-2) x^{2}+12(c-3) x^{3}-2(c+6) x^{4} & =0 \\ 36 c-6\left(3 c^{2}-4 c+72\right) x+18\left(c^{2}-6 c\right) x^{2}-4\left(c^{2}+12 c+20\right) x^{3} & =0\end{cases}
$$

has the solution $c=x=0$ evidently. Let $x \neq 0$ and $x \neq 3$. From the first equation we get

$$
c=\frac{6\left(1-x^{2}\right)(x+3)}{x(x-3)^{2}}
$$

which satisfies the inequality $0 \leq c \leq 2$ only when $x \in\left[x^{\prime}, 1\right)$, where $x^{\prime} \approx 0.81244$. Now substituting (24) into the second equation of (23) we obtain the equation

$$
4 x^{6}+45 x^{5}-333 x^{4}+162 x^{3}-486 x^{2}+945 x-81=0,
$$

which has the unique solution in $(0,1)$, namely $x^{\prime \prime} \approx 0.089756<x^{\prime}$. Thus $g$ has no critical point in the interior of $\Delta_{2}$.

Summarizing, from par D it follows that

$$
\left|T_{4,1}(f)\right|=\frac{1}{82944}\left(4-c^{2}\right)^{3} F(c, x, y, \theta, \psi) \leq \frac{1}{82944} \cdot 4^{3} \cdot 1296=1 .
$$

It is clear that equality for the upper bound in (12) holds for the identity function, and for the lower bound for the function (9).

\section{Compliance with ethical standards}

Conflict of interest The authors declare that there is no conflict of interest.

Open Access This article is licensed under a Creative Commons Attribution 4.0 International License, which permits use, sharing, adaptation, distribution and reproduction in any medium or format, as long as you give appropriate credit to the original author(s) and the source, provide a link to the Creative Commons licence, and indicate if changes were made. The images or other third party material in this article are included in the article's Creative Commons licence, unless indicated otherwise in a credit line to the material. If material is not included in the article's Creative Commons licence and your intended use is not permitted by statutory regulation or exceeds the permitted use, you will need to obtain permission directly from the copyright holder. To view a copy of this licence, visit http://creativecommons.org/licenses/by/4.0/.

\section{References}

1. Ali, M.F., Thomas, D.K., Vasudevarao, A.: Toeplitz determinants whose elements are the coefficients of analytic and univalent functions. Bull. Aust. Math. Soc. 97, 253-264 (2018)

2. Carathéodory, C.: Über den Variabilitatsbereich der Koeffizienten von Potenzreihen, die gegebene werte nicht annehmen. Math. Ann. 64, 95-115 (1907) 
3. Cho, N.E., Kowalczyk, B., Lecko, A.: Sharp bounds of some coefficient functionals over the class of functions convex in the direction of the imaginary axis. Bull. Aust. Math. Soc. 100, 86-96 (2019)

4. Cudna, K., Kwon, O.S., Lecko, A., Sim, Y.J., Śmiarowska, B.: The second and third-order Hermitian Toeplitz determinants for starlike and convex functions of order $\alpha$. Bol. Soc. Mat. Mexicana 26, 361375 (2020)

5. Duren, P.L.: Univalent Functions. Springer, New York (1983)

6. Goodman, A.W.: Univalent Functions. Mariner, Tampa, Florida (1983)

7. Kowalczyk, B., Kwon, O.S., Lecko, A., Sim, Y.J., Śmiarowska, B.: The third-order Hermitian Toeplitz determinant for classes of functions convex in one direction. Bul. Malays. Math. Sci. Soc. 43, 31433158 (2020)

8. Libera, R.J., Zlotkiewicz, E.J.: Early coefficients of the inverse of a regular convex functions. Proc. Am. Math. Soc. 85(2), 225-230 (1982)

9. Libera, R.J., Zlotkiewicz, E.J.: Coefficient bounds for the inverse of a function with derivatives in $\mathcal{P}$. Proc. Am. Math. Soc. 87(2), 251-257 (1983)

10. Pommerenke, C.: Univalent Functions. Vandenhoeck \& Ruprecht, Göttingen (1975)

11. Study, E.: Vorlesungen über ausgewählte Gegenstände der Geometrie, Zweites Heft; Konforme Abbildung Einfach-Zusammenhängender Bereiche. Druck und Verlag von B.G. Teubner, Leipzig und Berlin (1913)

Publisher's Note Springer Nature remains neutral with regard to jurisdictional claims in published maps and institutional affiliations. 